\title{
MOTIVACIÓN PARA EL APRENDIZAJE DE ESPAÑOL EN UNA UNIVERSIDAD FRANCESA FRONTERIZA
}

\author{
María Ángeles Goicoechea Gaona \\ Universidad de La Rioja
}

\begin{abstract}
RESUMEN: Este artículo centrado en conocer acerca de la motivación del alumnado de la Université de Pau et des Pays de l'Adour por el estudio de español como lengua extranjera se ha servido de la metodología cualitativa (entrevistas abiertas y su posterior análisis). Se han obtenido los siguientes resultados: Existe alta motivación entre el alumnado de dicha universidad por el español, debido a las actitudes y motivaciones del profesorado, y a la cercanía y coincidencia entre la cultura francesa y la española, entre otras. Respecto de las dificultades se señalan el escaso vocabulario relacionado con la vida cotidiana, la complejidad de la conjugación verbal y de la pronunciación y una limitada comprensión de los hablantes autóctonos. Por todo ello, se ha documentado la demanda de afianzar la enseñanza de las competencias comunicativas orales. De esta investigación se puede inferir que existe una importante motivación intrínseca en el alumnado de todas las facultades de esta universidad.
\end{abstract}

PALABRAS CLAVE: Motivación por el rendimiento, lenguas extranjeras, enseñanza superior, investigación cualitativa.

\section{MOTIVATION FOR THE LEARNING OF SPANISH IN A FRENCH UNIVERSITY ON THE BORDERS}

ABSTRACT: This paper examined the motivation of the University of Pau students to learn Spanish as a foreign language. Qualitative methods have been used such as the analysis of open-ended interviews. The research findings show that students at the University of Pau are highly motivated to learn Spanish because of their teachers' attitudes and the cultural similarities between Spain and France. Our studies revealed some difficulties of the French students related to the pronunciation, vocabulary used in daily life, Spanish verb conjugation and listening comprehension of native speakers. As a result, 
the development of oral communication skills is recommended. The report concludes that there is an intrinsic motivation (to learn Spanish) among the University of Pau students at each Faculty.

KEYWORDS: Motivation of accomplishment, foreign languages, Higher Education, qualitative research.

Recibido: 03/02/2016

Aceptado: 11/05/2016

Correspondencia: María Ángeles Goicoechea Gaona, Universidad de La Rioja, Departamento de Educación, C/ Luis de Ulloa, Edificio Vives, 26004 Logroño (La Rioja). Email: angeles.goicoechea@unirioja.es.

Me encanta el SR. T. porque es totalmente distinto por... Pero creo que tiene esta sensibilidad literaria que a mí me gusta y también no te dice: "Yo sé todo, ni vosotros nada". Y eso es importantísimo. Te dice: "Yo no sé, tú me vas a decir". Y creo que es una... [E': es una virtud.] Sí. (2.m.e)

La finalidad de este trabajo es conocer acerca de la motivación del alumnado de la Université de Pau et des Pays de l'Adour (UPPA) por el estudio de español como lengua extranjera. La relevancia de este tema radica en el hecho de que actualmente los intereses comerciales marcan la primacía en el estudio de las lenguas de servicio que impone el mercado sobre las lenguas de cultura, como el español y el francés, por ejemplo.

Pau es una ciudad del Departamento Pyrénées Atlantique situada junto a la región de Béarn, cercana a los Pirineos, frontera natural con España. Su vinculación con nuestro país es el resultado de que en el pasado, dada la proximidad geográfica de los dos estados, se haya acogido a inmigrantes españoles desplazados por motivos políticos (la Guerra de Argelia o la Guerra Civil española) y sociales (personas en busca de trabajo). Su universidad acoge a estudiantes de las regiones de Béarn, del Pays Basque y de Hautes Pyrénées que viven en municipios cercanos a la frontera y cuyos progenitores tienen contactos laborales con personas españolas. En este contexto se comprende el interés por la lengua española, tanto como lengua de cultura como de negocios.

Del objetivo general se derivan otros más concretos, como averiguar las razones que motivan la elección del español como lengua extranjera y establecer si existen diferencias significativas entre el estudiantado de las distintas titulaciones que la elige.

\section{FUNDAMENTACIÓN TEÓRICA}

En momentos en los que en Europa se privilegia la construcción económica sobre la construcción cultural y las lenguas propias de cada país parecen correr el riesgo

1. La E se refiere a la entrevistadora. 
de quedar relegadas a usos cotidianas e íntimos; cuando, de la mano del proceso de la globalización, las denominadas lenguas de servicio, exclusivamente vinculadas al poder económico, se nos imponen e invaden los lugares de trabajo y formación, es necesario hacer hincapié en lo que Rastier (2013) ha denominado la educación cultural de la unidad europea. En este contexto el autor aboga por una valoración de los aspectos culturales inherentes a toda lengua, frente a un uso limitado a las transacciones comerciales, que no hace otra cosa que empobrecer la lengua en cuestión y condenar a los hablantes al progresivo debilitamiento de su identidad. El autor defiende las lenguas de cultura ligadas a comunidades con historia propia como medio para preservar la unidad de cultura gracias a la diversidad de las lenguas.

En el ámbito de la educación, la motivación para el aprendizaje escolar ha sido estudiada y clasificada en dos tipos por varios autores (Tapia, 2005; Minera, 2009):

1. M. extrínseca: es la que se produce a través de hechos o deseos que proceden del exterior de la persona, por ejemplo, cuando padres, profesores o educadores aportan razones y animan al educando a estudiar. Este tipo de motivación normalmente se produce en edades tempranas.

2. M. intrínseca: aquella que se origina en el interior del individuo que le lleva a elegir un aprendizaje. Muchas veces se llega a esta motivación, porque en etapas evolutivas anteriores ha habido personas significativas que le han animado a realizar determinadas actividades. "... es gratificante en sí mismo" (Minera, 2009, p. 60).

En el caso concreto de la lengua extranjera, un estudio de Gadner y Temblay (1994, citado por de la Morena, Sánchez y Fernández, 2011) diferencia dos tipos de motivación, relacionados con los anteriormente descritos y, de algún modo, complementarios:

1. M. integrativa: "es un constructo formado por tres características: las actitudes hacia el aprendizaje del idioma (afecto), el deseo de aprender el idioma (querencia) y la intensidad emocional (esfuerzo). Todos ellos vinculados a la motivación intrínseca" (de la Morena, Sánchez y Fernández, 2011, p. 226). Dentro de este tipo se especifica la motivación sociocultural que "se asocia a intereses de tipo sociocultural, dirigidos a la comunidad de la lengua objeto (la gente, la cultura y otros aspectos afines) sin necesariamente perseguir el objetivo de integrarse en la comunidad meta" (Minera, 2009, p. 61).

2. M. instrumental: "las motivaciones prácticas que dirigen el aprendizaje del alumno, más vinculada a la motivación del logro" (de la Morena, Sánchez y Fernández, 2011, p. 226) y que, en palabras de Minera (2009), poseen un propósito utilitario.

Estos tipos de motivaciones siguen siendo estudiados y forman parte de la fundamentación en investigaciones aplicadas al aprendizaje de una lengua extranjera (LE), estableciendo la diferencia entre estos dos tipos de la siguiente manera:

...en el estudio de una LE se ha hecho una división entre una motivación de carácter integrador, es decir, se estudia la LE porque de algún modo el individuo se siente atraído y desea integrarse en la comunidad y sociedad que habla esa lengua y de la que quiere formar parte, y la motivación instrumental, es decir, aquella que está 
alimentada por razones prácticas concretas y materiales: conseguir un trabajo con mayor facilidad, promocionar laboralmente o tener mayores expectativas de salida al exterior. (Palacios, 2014, p. 22)

En este sentido se encaminan algunas de las conclusiones de este estudio. Otras investigaciones hacen hincapié en el vínculo que se establece entre afectividad y motivación en el aprendizaje de una lengua extranjera, pues manifiestan que

...la adquisición de una nueva lengua genera emociones específicas susceptibles de potenciar o bloquear los procesos de aprendizaje. Esas emociones están vinculadas al hecho de que un idioma extranjero exige del aprendiz conductas fónicas, gestuales, comportamientos culturales y hábitos de pensamiento que cuestionan las de su propia lengua materna, afectando a su identidad. (Rodríguez Pérez, 2012, p. 406)

Como se aprecia en el testimonio de la cita que abre este artículo, la actitud del profesorado para contribuir a que el alumnado supere estos bloqueos emocionales y se muestre favorable al aprendizaje es fundamental.

La motivación es un factor prioritario en la educación y en este sentido afirmamos con Arnold y Fonseca que "conocer las necesidades y motivaciones de los alumnos es un primer paso imprescindible para que se dé un proceso de enseñanza/aprendizaje eficaz, basado en lo que es relevante para 'estos' alumnos en 'esta' aula" (2004, pp. 52-53).

\section{Metodología}

La metodología elegida para el desarrollo de esta investigación es la cualitativa, siendo la técnica empleada para la obtención de datos la entrevista semiestructurada y el análisis de contenido de los textos generados a partir de las entrevistas.

Los métodos cualitativos se caracterizan por ahondar en las razones emitidas por los participantes. Muchos de los estudios desarrollados con este tipo de metodología, por los recursos que requieren y por la profundidad que pretenden (Ruiz, 2007; Gibbs, 2012), se realizan con una población más limitada y mucho más reducida que en los de corte cuantitativo.

La metodología cualitativa posee una serie de características (Ruiz, 2007, p. 23) que se cumplen en esta investigación como son la intención de captar el significado de los procesos; el uso del lenguaje conceptual y simbólico, más que numérico; la recopilación de datos flexible; ser más inductiva que deductiva; y ser holística y concretizadora, es decir, no pretende generalizar los resultados, sino mostrarlos en su contexto y proceso.

De entre las técnicas de recogida de información cualitativa, en este estudio se ha optado por la entrevista que "es una conversación que tiene una estructura y un propósito determinados por una parte: el entrevistador" (Kvale, 2011, p. 30). En concreto se ha optado por la entrevista semiestructurada de preguntas abiertas con un guion orientativo.

Para tratar los datos obtenidos se ha realizado un análisis de contenido que es "una estrategia que trata de encontrar unidades de significado temático dentro de un texto que permitan hacer algún tipo de inferencia sobre procesos que afectan a los participantes que produjeron dichos textos" (León y Montero, 2015, p. 568). 
Estas técnicas de investigación cualitativa han procurado resultados que ahondan en las razones de los estudiantes para elegir una lengua extranjera determinada, en función de las necesidades, expectativas, dificultades o facilidades que prevén y de sus propias características personales y académicas.

\section{Los informantes}

Con el fin de recopilar datos en los que fundamentar los resultados de esta investigación se han mantenido reuniones breves con el profesorado de lengua española de la Universidad de Pau de distintas titulaciones para seleccionar a quienes pudieran participar. Se ha tratado de que la muestra sea representativa, escogiendo alumnos que cursan estudios clasificados tradicionalmente en "letras" y "ciencias".

La elección de las personas participantes de esta investigación ha seguido criterios diversos, teniendo presente la capacidad comunicativa del estudiante seleccionado para expresarse en lengua castellana y su disponibilidad temporal. Las categorías tenidas en cuenta para designar a quienes participaron han sido, por una parte, el sexo y, por otra, el área de formación, es decir, la facultad y/o titulación.

La población objeto del estudio está conformada por un alumno y una alumna de lengua española de cada una de las facultades y/o titulación, con una excepción que se explica a continuación:

1. Dos de la Faculté de Droit, Économie et Gestion (abreviando Faculté de Droit): un estudiante de derecho y una alumna de economía.

2. Tres de la Faculté de Sciences et Technologies (Faculté de Sciences): un chico y una chica que estudian para ser profesores de educación primaria. Además se entrevistó a una alumna más de ciencias con perfil tecnológico, al descubrir que la orientación profesional de los otros no iba a ser las ciencias exactas sino la docencia.

3. Dos alumnos de la Licence d'Espagnol de la Faculté d'Arts, Lettres, Langues, Sciences Humaines et Sociales (Faculté de Lettres): un hombre y una mujer.

4. Dos alumnos de la Faculté de Lettres, del resto de titulaciones, exceptuando el español: un chico y una chica.

En la selección de los y las participantes se ha tenido en cuenta el sexo y se ha buscado el equilibrio entre unas y otros, siendo 5 de ellas mujeres y 4 hombres, con edades que fluctúan entre los 18 y 45 años, encontrándose la mayoría entre la edad mínima y los 22 años.

\section{La técnica}

La entrevista abierta y en profundidad es la técnica elegida para el desarrollo de esta pesquisa de carácter cualitativo. A través de este instrumento se recogen, en primer lugar, datos personales como son: sexo, facultad, estudios, curso y edad. Después se establecen en 10 preguntas abiertas como, por ejemplo, ¿qué ventajas o facilidades encuentras para aprender esta lengua extranjera?, ¿hay algunas que sean distintas al aprendizaje por ejemplo del inglés? El orden de las cuestiones no es en 
todos los casos el mismo, pues puede darse el caso de que un informante responda en el desarrollo de su discurso a alguna de las cuestiones planteadas sin necesidad de preguntar directamente.

Esta técnica, además de permitir profundizar y ahondar en las cuestiones que sobre la marcha van surgiendo por parte de la persona entrevistada o de la entrevistadora, tiene la ventaja de poder apreciar el nivel de adquisición de la lengua castellana, objeto secundario, pero fundamental de este estudio. Las facilidades y dificultades que presenta un estudiante ante el aprendizaje de una segunda lengua extranjera se observan, no solo en las respuestas que emite el informante, sino en la capacidad para expresarse de manera oral.

Por este motivo, las entrevistas se realizan en español, lengua materna de la investigadora y conocida por toda la población estudiada, porque su uso facilita la tarea de la primera, le permite captar sutilezas de discursos encubiertos y la transcripción posterior. En el transcurso de cada entrevista se recomienda al alumnado hablar en francés, cuando tengan dificultades para hacerlo en castellano, pues es una lengua que también conoce la entrevistadora.

Cada entrevista se administra de forma individual por tres motivos fundamentales: porque es más fácil establecer un vínculo de confianza en poco tiempo con una sola persona; para evitar que un estudiante con alto dominio del castellano o con una personalidad abierta monopolice la conversación; para facilitar la transcripción y la comprensión de todo el discurso, puesto que, si hay varios informantes, sus voces pueden superponerse.

\section{El procedimiento y análisis}

Las entrevistas tienen duración variable, siendo la más larga de una hora. Estas se graban en formato digital y se transcriben con posterioridad. Para la identificación las personas participantes se asignan códigos con el siguiente formato:

(número de entrevista.sexo.titulación y/o facultad a la que pertenece) ${ }^{2}$

Los códigos correspondientes a las nueve personas entrevistadas son los siguientes: (1.m.l), (2.m.e), (3.h.e), (4.m.d), (5.h.d), (6.h.c), (7.m.c), (8.m.c) y (9.h.l).

Este sistema de codificación aporta dos datos importantes para nuestro posterior análisis, como es el sexo y la facultad del informante.

La propia investigadora realiza las nueve entrevistas y las transcribe literalmente, añadiendo entre paréntesis manifestaciones de lenguaje no verbal. Durante este proceso tan solo se corrigen algunos errores frecuentes, repetidos por prácticamente la totalidad de los entrevistados cuya lengua materna es el francés, entre los que destacamos:

- Confusión del verbo "ser" y "estar" ("el profesor está bueno").

- Uso incorrecto de las preposiciones ("ir en España").

2. El número corresponde al orden de realización de la entrevista. El sexo es una " $m$ ": mujer y una "h": hombre. La titulación y/o facultad se marca con una "c": Faculté de Sciences, una "d": Faculté de Droit, una "l": Faculté de Lettres y una "e": Licence d' Espagnol. 
- Utilización inadecuada de algún tiempo verbal como pretérito perfecto simple y el pretérito imperfecto ("estudiamos" por "estudiábamos").

- Olvido de pronombre reflexivo en algunos verbos.

- Uso literal de léxico francés debido a su similitud fonética o gráfica con el español ("timide").

El análisis de estas nueve entrevistas se presenta como una lectura profunda de las mismas, es decir, se trata de "reflexionar teóricamente sobre temas específicos de interés, redactar una interpretación..." (Kvale, 2011, p. 151).

Además de resaltar aquellos errores más frecuentes en el uso del castellano en este alumnado francés, como se ha puesto de manifiesto arriba, se hizo un análisis de contenido atendiendo a estas dos categorías:

- Motivación intrínseca: Necesidades de comunicación y razones que conducen a la elección del español.

- Motivación extrínseca.

\section{Resultados del anÁlisis: condicionamientos en la elección de la LENGUA CASTELLANA}

\section{Motivación intrínseca: Necesidades de comunicación y razones que conducen a la elección del español}

De los participantes, solo uno ha nacido en España en una familia mixta (padre español y madre francesa), aunque desde pequeño vive en Francia, por lo que su lengua materna y de uso cotidiano es la francesa, con su padre habla en castellano. Otra estudiante tiene ancestros (tatarabuelos) españoles.

Todos los entrevistados mantienen lazos con España por diversos motivos: comerciales -dos viven junto a la frontera-; familiares -tres tienen algún familiar cuya primera o segunda residencia está en este país- y de ocio -nueve lo eligen como destino de vacaciones-. Es decir, que el cien por cien de las personas entrevistadas conocen España por haber realizado viajes de ocio (vacaciones, visitas, etc.), pedagógicos (estancias de estudios, intercambios o excursiones escolares) o laborales (trabajo).

En las familias de varios de los estudiantes, uno o ambos progenitores sin ser de origen español, habla bien la lengua castellana:

Mi padre no lo habla muy bien, pero habla corriente. Y mi madre habla bastante bien, porque ella -cómo se dice- ella siempre iba de vacaciones allí, tenía a todos sus amigos y sí, entonces ella habla bastante bien. Y yo hablo un poco español, porque tengo también mis amigos allí y tengo que hablar con ellos, porque... (7.m.c)

Los adultos mencionados que dominan la competencia lingüística porque frecuentan España por los mismos motivos arriba señalados, influyen en sus hijos, pues como defiende Rodríguez: "[...] el ambiente socio-cultural y los aspectos que éste incumbe pesan más en la adopción de una segunda lengua" (2010, p. 236). Así afirmamos que la mayoría de estos estudiantes están predispuestos favorablemente hacia el estudio de esta lengua extranjera por sus experiencias familiares y vitales. 
Las razones por las que el alumnado de la UPPA, independientemente de la titulación que cursen, se decide a estudiar el español como lengua extranjera, son diversas. Es preciso destacar que algunos centros del sur de Francia -a tenor de lo que manifiestan algunas personas entrevistadas- ofrecen español como primera lengua extranjera en lugar de la inglesa, posiblemente porque el castellano es un medio de relación con el país vecino, al tratarse de una región fronteriza.

Todos y todas las entrevistadas iniciaron estos estudios antes de ingresar en la universidad. Para siete fue su primera elección y en dos de estos casos, lo iniciaron a la vez que el inglés, ya que a los alumnos que obtienen buenas calificaciones, se les permite hacerlo. Los otros dos restantes optaron por el español como segunda lengua extranjera.

La edad de inicio del estudio de la lengua castellana es temprana, ya que oscila entre 9 y 12 años, si bien uno de los entrevistados manifiesta haber comenzado a los 5 o 6 años.

La oferta académica en materia de lenguas extranjeras que presentan los centros educativos guarda relación con el poder económico y el estatus social y político que estas representan. La lengua española en Francia no es la más reconocida dentro de su sistema educativo, siendo el inglés la de estudio más común; además, existen otras, de menor uso a nivel mundial, pero mejor consideradas como nos muestra este testimonio:

[...] he visto que había una jerarquía de las lenguas con respecto a un nivel. Por ejemplo, algunos alumnos eligen el idioma, porque sabe, por ejemplo, que van a encontrarse en una clase que tiene un nivel más alto. Por ejemplo, alemán es la lengua de los... sí, de los más fuertes en el cole. Y entonces, el idioma en los colegios y en los institutos, me parece que hacen una, una... cómo decir, una división y que participan en una casi discriminación escolar. [E: O sea, una jerarquización entre buenos y malos.] Sí. Y por ejemplo, los que... bueno las clases con los alumnos de nivel más bajo eran clases de español. [E: Más alto el alemán, luego estaba el inglés y después el español.] Sí, sí, alemán o ruso, después inglés y después español. Y me encontraba en clases muy, bueno, que tenían una reputación mala. (1.m.l)

A la dificultad que conlleva el estudio de una lengua se suma tener que decidirse por una u otra, asumiendo que algunas como la española no gozan de la mejor reputación y que esta opción puede derivar al estudiante a un grupo-clase de nivel medio o medio bajo.

A pesar de la constatación arriba señalada, esta situación puede cambiar en determinadas zonas de Francia por el efecto fronterizo que determina la elección de lenguas no tan apreciadas, en nuestro caso, el español:

1. Proximidad física: vivir en el Pirineo, frontera natural con España, une y separa al mismo tiempo. Une porque las relaciones comerciales o sociales se dan, pero separa por las diferencias políticas, en las costumbres, en la lengua, etc.

Porque vivo al lado de España, al lado de la frontera. [E: ¿Dónde?] En (nombre del pueblo), al lado de Jaca. Y mi padre es agricultor y trabaja mucho con españoles, entonces... (4.m.d)

2. Proximidad simbólica: existen rasgos comunes o semejanzas entre la cultura e idioma de esta zona del sur de Francia, el patois u occitano, y la aragonesa en 
España. La frontera, división política, por sí sola no ha impedido los encuentros culturales producidos a lo largo de la historia y en el presente.

Lo decidí, porque vengo del sudoeste de Francia y se hablaba el patois, el Occitan en mi familia. No sé, mis padres no hablaban mucho, lo entendían, pero mis abuelos lo hablaban todos los días y yo oía este idioma todo el tiempo.

Y creo que, ahora, creo que he elegido este idioma por eso, porque me sonaba a algo que conocía ya, porque creo que hay muchos puntos comunes. (2.m.e)

Prácticamente la totalidad de las personas entrevistadas muestran esa cercanía en el parecido que existe entre la lengua francesa y la española:

Ya, primero porque mi prima es española (ríe), vive en Zaragoza y desde pequeños hablamos un poco español y como España es el país (que está muy) cerca, es fácil ir a España, escuchar a la gente y sobre todo porque es un idioma que se parece mucho al francés. Pues no es muy (tan) difícil como el inglés. [...] Porque las palabras se parecen mucho, porque al final hay que cambiar un poco la letra "e", "a" (ríe) y podemos entendernos. (6.h.c)

3. Proximidad parental, pues algunos tienen familia o amigos que reside de forma permanente o discontinua en España, como en la cita anterior y en la siguiente:

Es un idioma que me gusta sin más, que por ejemplo el inglés. Y es que estamos muy cerca de España. Y que tengo un poco de familia allá, bueno, francesa. No tengo origen españoles, pero tengo familia francesa que fue a España, pero sobre todo a Cataluña.

Y es eso que me gusta más y no sé para, no sé, no sé, es el idioma que es más... pero la cultura también. (1.m.l)

Este testimonio hace hincapié en el origen francés de esta estudiante, que reitera desde el inicio de la entrevista, y contrasta con otro entrevistado que no tiene problemas en decir que su tío se casó con una española, reconociendo así el mestizaje.

Otro argumento que se aporta en la cita de arriba y en otras entrevistas, fundamentalmente entre quienes han elegido cursar la Licence $d^{\prime} E s p a g n o l$, es el gusto por la cultura hispánica. Este aspecto significa una motivación intrínseca o integrativa, puesto que son adultos que han elegido libremente:

Sabía que quería cursar lenguas extranjeras, pero al principio quería ser profe de inglés, y después he descubierto el español, la lengua, y me ha atraído mucho la cultura, la lengua, la historia también, porque está muy relacionado con América Latina. Y esto me atrae también mucho. (3.h.e)

\section{Motivación extrínseca}

La influencia del primer profesor de una lengua extranjera, en esos momentos fuente de motivación extrínseca, también puede ser decisiva a la hora de continuar aprendiéndola después:

Porque mi profesor en el colegio era muy bueno y me dio envidia para conocer y aprender español. (4.m.d) 
En términos mercantilistas, a esta alumna de economía así como a otros estudiantes de derecho y ciencias les hubiera resultado más rentable a nivel académico y profesional aprender inglés, pero la motivación contagiada por el profesorado ha sido decisiva en su elección.

La actitud positiva del profesor repercute en el buen clima de clase que "es básico para promover las relaciones positivas" (Medina, 2009, p. 611), idea que comparte un alumno, e imprescindible para tener deseos de aprender. Reducir la distancia entre docente y discentes, construyendo juntos un ambiente cordial que anime a preguntar, cuestionar y mostrarse como son:

Sí eso. Pues es muy importante que en un curso de lengua que haya una proximidad del profesor con el alumno, (más) que en otras materias (que) no es muy importante, solo que aprender, solo que... y si no quieres participar, no participas... Pero la proximidad con el profesor permite mucho más participar y... [...] "yo no soy un profesor, soy una persona como otra, con quien puedes hablar". (5.h.d)

El estudio de una lengua requiere de la práctica, por eso si no se dan las condiciones suficientes de confianza, respeto y motivación, el alumnado no se sentirá alentado para cooperar:

... la motivación y las actitudes son variables afectivas que no solamente favorecen el aprendizaje de los elementos lingüísticos de la lengua objeto (LO), sino que influyen en la perseverancia del aprendizaje y en el deseo de interactuar con la comunidad lingüística objeto, así como de comunicarse en dicha lengua. (Minera, 2009, p. 62)

Un aspecto más superfluo, pero que sin duda también actúa como motor para aprender esta lengua, es el aprecio del estilo de vida español:

La manera de vivir (ríe), muy tranquila... [E: ¿Muy tranquila en España?] Yo creo que la gente en España es muy tranquila y porque, no sé, porque viven más tarde que en Francia, hacen mucho la fiesta y no sé (ríe todo el tiempo). (6.h.c)

Este gusto por la forma de vida en España es reconocida por varios de los entrevistados.

\section{Discusión}

Al inicio de la investigación partíamos de ideas preconcebidas equivocadas como que el alumnado de la Faculté de Lettres habla mejor castellano que los de Sciences o Droit. Si bien el alumnado de la primera facultad posee un alto grado de conocimiento de esta lengua, con un amplio vocabulario y mayor nivel en la competencia lectora y escrita, el alumnado de Sciences y Droit tiene un dominio de la competencia oral elevado. No se han encontrado diferencias significativas al entrevistar a los de unas y otras titulaciones, pues el nivel de comprensión y expresión de prácticamente la totalidad de entrevistados es adecuado a su nivel universitario. Creíamos que la orientación hacia el área de conocimiento de la lengua otorgaba una mayor motivación hacia el aprendizaje de lenguas extranjeras, sin embargo hemos comprobado que en la motivación influyen otros factores que afectan por igual a todo el alumnado con independencia de la titulación que cursen. 
Los tipos de motivación referidos al inicio de este trabajo, descritos por de la Morena, Sánchez y Fernández (2011, p. 226), se aprecian en los discursos de los entrevistados y nos llevan a afirmar que se da un alto grado de motivación integrativa en quienes estudian para ser profesores de educación elemental o media; en menor medida en los que utilizarán de forma tangencial la lengua castellana en secundaria. Todas y todos las entrevistados manifiestan una gran cercanía y atracción hacia la lengua y cultura hispánica, lo que les mueve a desear aprenderla y a esforzarse para lograrlo.

En esta misma línea se encuentra una de las conclusiones de otra investigación realizada en torno al aprendizaje de una lengua extranjera:

Uno de los factores que contribuyen más poderosamente a fomentar, mantener o generar la motivación y las emociones positivas hacia el proceso de enseñanza aprendizaje de un idioma es el contacto directo de los alumnos y de los profesores con el país de la lengua extranjera. (Rodríguez Pérez, 2012, p. 406)

También se aprecia la motivación instrumental en todos los entrevistados que tienen interés por conseguir buenos resultados en esta disciplina y dominar las capacidades lingüísticas que les permitan utilizar los conocimientos en la práctica.

Ateniéndonos a la concepción clásica de motivación intrínseca y extrínseca, los resultados de este estudio se inclinan hacia la primera. El cien por ciento del alumnado entrevistado tiene interiorizado el deseo de aprender y ha elegido libremente la materia de español. Dos de los alumnos de ciencias además han decidido cambiar su curso por otro más completo y difícil de la Faculté de Lettres, por ejemplo. Sintetizando, sienten una fuerte motivación intrínseca.

También encontramos la motivación extrínseca en ese deseo generalizado por comunicarse con personas de otras culturas, en este caso, de habla castellana. De este modo los jóvenes hablantes actúan atraídos por los rasgos de cultura e identidad que las lenguas conllevan.

Se observa que la personalidad del entrevistado (persona tímida, se ríe nerviosamente o se muestra silenciosa) puede hacerle parecer inseguro y con poco dominio del idioma. Percepción que cambia a lo largo de la entrevista cuando la confianza generada se traduce en seguridad y tranquilidad. Lo mismo ocurrirá en el aula cuando el profesorado logre transmitir confianza, lo que repercutirá positivamente en la motivación y el aprendizaje.

\section{REFERENCIAS BIBLIOGRÁFICAS}

Arnold, J. y Fonseca, M. C. (2004). Reflexiones sobre aspectos del desarrollo de la competencia comunicativa oral en el aula de español como segunda lengua (4560). En S. Ruhstaller y F. L. Berguillos, La competencia lingüística y comunicativa en el aprendizaje del español como lengua extranjera. Madrid: Edinumen.

Gibbs, G. (2012). El análisis de datos cualitativos en Investigación Cualitativa. Madrid: Morata.

De la Morena, M., Sánchez, A. y Fernández, M. P. (2011). Diferencias en los niveles de motivación por sexo, curso y tipo de centro educativo en el aprendizaje estratégico de la lengua extranjera. ARBOR Ciencia, Pensamiento y Cultura, 187(Extra 3), 225-230. 
Kvale, S. (2011). Las entrevistas en Investigación Cualitativa. Madrid: Morata.

León, O. G. y Montero, I. (2015). Metodología de investigación en psicología y educación. Las tradiciones cuantitativa y cualitativa. Madrid: McGraw-Hill.

Medina Rivilla, A., Rodríguez Diéguez, J. L. y Sevillano García, M. L. (Coords.) (2009). Diseño, desarrollo e innovación del currículum en las instituciones educativas. Tomo II. Madrid: Universitas.

Minera Reyna, L. E. (2009). El papel de la motivación y las actitudes en el aprendizaje de ELE en un contexto de enseñanza formal para adultos alemanes. Revista Lebrija de Lingüística Aplicada, 6(3), 58-73.

Palacios, I. M. (2014). La motivación en el aprendizaje de una lengua extranjera. ¿Qué podemos hacer con alumnos desmotivados? Mosaico. Revista para la promoción y apoyo a la enseñanza del español, 32, 22-28.

Rastier, F. (2013). Apprendre pour transmettre. L'éducation contre l'idéologie managériale. Paris: PUF.

Rodríguez Díez, S. (2010). Tendencias actuales en el aprendizaje-adquisición de las lenguas extranjeras: la didáctica del Francés Lengua Extranjera (FLE). Foro de Educación, 12, 233-253.

Rodríguez Pérez, N. (2012). Causas que intervienen en la motivación del alumno en la enseñanza-aprendizaje de idiomas: el pensamiento del profesor. Didáctica. Lengua y Literatura, 24, 381-409.

Ruiz Olabuénaga, J. I. (2007). Metodología de la investigación cualitativa. Bilbao: Universidad de Deusto.

Tapia, A. (2005). Motivación para el aprendizaje: la perspectiva de los alumnos. En Ministerio de Educación y Ciencia. La Orientación escolar en los centros educativos (pp. 209-242). Madrid: MEC.

Yule, G. (1998). El lenguaje. Cambridge: Cambridge University Press. 